\title{
Dissociating nociceptive modulation by the duration of pain anticipation from unpredictability in the timing of pain
}

\section{A R T I C L E I N F O}

\section{Article history:}

Accepted 21 September 2008

Available online $\mathrm{xxxx}$

\section{Keywords:}

EEG

LORETA

Prediction

Nociception

Attention A B S T R A C T
Objective: Waiting longer to receive pain increases its perceived unpleasantness by inducing 'dread'.
However, it is not clear how unpredictability in the timing of the impending pain stimulus interacts with
dread and whether the two factors show differential effects on the neural generators of the pain-evoked
response.
Methods: We manipulated the duration of anticipation of laser-induced pain independently of unpredict-
ability of stimulus delivery timing, to observe the relative effect on P2 amplitudes of the laser-evoked
potential (LEP) response and its estimated sources.
Results: Subjects ( $n=12$ ) reported increased pain ratings after longer pain anticipation, irrespective of
unpredictability in the timing of stimulus delivery. By contrast, unpredictability in stimulus timing
increased the amplitude of the P2 irrespective of anticipation duration. The modulation of P2 amplitude
by unpredictability was localized to midcingulate cortex (MCC) and ipsilateral secondary somatosensory
(S2) areas. Greater anticipation duration increased activity in a hippocampal-insula-prefrontal network
but not in MCC areas.
Conclusions: Distinct neural networks contribute to the P2 and are differentially affected by pain antici-
pation duration and unpredictability in stimulus timing.
Significance: ERP research into dread should be careful to appreciate the neural generators of pain-evoked
responses and their potential modulation by unpredictability.
() 2008 International Federation of Clinical Neurophysiology. Published by Elsevier Ireland Ltd. All rights
reserved.

\section{Introduction}

The experience of pain integrates sensory information about the intensity, timing and location of the stimulus with cognitive and affective information (Melzack and Wall, 1965; Craig, 2003). Pain is mediated through a network of distributed areas in the brain, the pain matrix (Melzack, 2001). The pain matrix consists of the medial pain system associated with processing the affective and motivational aspects of pain and the lateral pain system responsible for encoding sensory-discriminative information and motor coordination responses (Jones et al., 2003; Vogt, 2005). Although these two pain systems can be differentiated (Kulkarni et al., 2005; Rainville et al., 1999), it is clear that changes in the sensory characteristics of pain modulate the affective value of the stimulus (Price, 2000).

One way in which the affective characteristics of pain can be modified is by altering the timing of the stimulus. As is common in dealing with unpleasant events, most people would choose to

\footnotetext{
* Corresponding author. Tel.: +44 (0)161 2064528

E-mail address: christopher.brown@manchester.ac.uk (C.A. Brown).
}

have a painful stimulus delivered quickly rather than wait for it (Berns et al., 2006). This phenomenon extends to choosing to have a more painful stimulus given sooner rather than wait for a less painful one (Berns et al., 2006). Behavioral models (Caplin and Leahy, 2001) assume this occurs because there is a cost to waiting, i.e., dread. Using functional magnetic resonance imaging (fMRI), the neural correlates of dread during anticipation of pain have been localized to key areas of the pain matrix including midcingulate cortex, primary and secondary somatosensory cortices, and posterior insula cortex. A further study utilized the temporal resolution of electro-encephalography (EEG) and magnetoencephalography (MEG) to specifically look into the effects of longer anticipation periods on the pain-evoked response (Hauck et al., 2007). It showed that longer pain anticipation durations increase the amplitude of the P2 component, which is known to be generated mainly from the midcingulate cortex (Bentley et al., 2003; Garcia-Larrea et al., 2003), in addition to augmenting pain experience. However, research has also revealed potential sources of the P2 in posterior parietal, medial temporal and anterior insular regions (Bentley et al., 2001; Valeriani et al., 2000). It is not clear which P2-generating brain regions contribute to the 
ncreased pain unpleasantness resulting from longer pain anticipation durations.

One factor that may interact with the length of anticipation and its associated dread is unpredictability in the timing of the painful event. Predictability in general determines the level of uncertainty with which a pain stimulus is anticipated, and may take the form of uncertainty about the intensity, location, timing or type of a stimulus. Predictability has an adaptive value, in that it allows organisms to develop behavioral control strategies, and like control, predictability can modulate the extent to which aversive stimuli induce stress and anxiety (Miller, 1981).

No study to date has controlled for the possible modulatory influences of unpredictability in stimulus timing when investigating the effects of anticipation duration on pain responses. Attention may be modulated by unpredictability in a way that modifies the pain-evoked response. The importance of attention in modulating the pain response is widely acknowledged (Buffington et al., 2005; Keefe et al., 2004; Bantick et al., 2002; McCaul and Haugtvedt, 1982; Hauck et al., 2007; Kulkarni et al., 2005). Both theoretical considerations and evidence suggests that that any kind of uncertainty (Dayan and Abbott, 2001; Brown et al., 2008), and specifically uncertainty in the timing of pain (Carlsson et al., 2006), modulates attention to the stimulus. Research using fMRI has shown that varying the unpredictability of stimulus timing causes differential brain responses in areas associated with attention and affective processing (Carlsson et al., 2006). Hence it is important to control as far as is practical for the effect of attention in order to accurately ascertain the effects of uncertainty and anticipation period duration on pain responses.

The aim of this study was to investigate the effects of both anticipation duration and unpredictability of stimulus timing on pain-evoked responses and perceived pain, whilst controlling for possible modulatory effects of attention. We manipulated the unpredictability in the timing of pain delivery, independently of the anticipation duration, allowing us to determine the main effects of each. We predicted that increasing the anticipation duration would increase the perceived painfulness of the stimuli, independently of their unpredictability. We expected this effect to be associated with increased pain-evoked responses in areas of medial pain system associated with pain unpleasantness, including midcingulate cortex. We further expected that manipulation of the unpredictability of pain would modulate attention-related activity in midcingulate cortex independently of anticipation duration.

\section{Methods}

\subsection{Subjects}

Twelve healthy, right-handed subjects, free of psychiatric, neurological, cardiovascular or autonomic disorders, participated in the study (mean age $21.25 \pm 2.0$ ). Subjects gave informed written consent, and the study was approved by Oldham Local Research Ethics Committee.

\subsection{Experimental procedure}

Laser heat stimuli of 150 ms duration and a beam diameter of $15 \mathrm{~mm}$ were applied to the dorsal surface of the subjects' right forearm using a $\mathrm{CO}_{2}$ laser stimulator. Between stimuli, the laser was moved randomly over an area $3 \mathrm{~cm} \times 5 \mathrm{~cm}$ to avoid habituation, sensitization or skin damage. $\widehat{\text { Subjects }}$ wore protective laser safety goggles during the experiment.

An initial psychophysics procedure was performed using a 0-10 sensory rating scale, which was anchored such that a level 0 indicated no sensation, level 4 indicated the pain threshold and a level
7 indicated moderately painful. Participants were told to regard 'moderately' painful as halfway between pain threshold and the maximum they could tolerate (which also corresponds to how the level 7 is defined on the scale - halfway between level 4 (threshold) and 10 (tolerance)). A ramping procedure was repeated three times to determine laser intensities rated as a level 7 , for each subject. Subjects' ratings of the intensity level were then tested through a series of laser pulses and the intensity levels were adjusted to achieve an appropriate level of sensation.

The start of each trial was indicated by the presentation of a visual stimulus on a computer screen. Participants were made aware that the laser stimulus would be delivered at either $3,6,9$, or 12 s and that there was an equal probability of any trial occurring. Three seconds was chosen as the minimum anticipation duration to remain consistent with our previous studies (Brown et al., 2008; Brown et al., in press; Brown and Jones, in press), in which we aimed to separate early and late anticipatory processes. By maintaining this separation for the shortest anticipation periods in the present study we ensured no overlap of early anticipatory processes and those related to pain processing. Multiples of $3 \mathrm{~s}$ were used for further conditions so that anticipation duration could be regarded as a linear variable in statistical analyses.

There were two experimental conditions, predictable and unpredictable, that differed according to the subject's knowledge of the timing of the laser stimulus. In both conditions a number was displayed in a blue triangle prior to the stimulus delivery, but only in the predictable condition did the number indicate the timing (in seconds) prior to the stimulus: numbers were displayed within a downwards-pointing triangle, which counted downwards starting at 3,6,9, or 12 (representing the number of seconds until stimulus delivery) until the delivery of the laser stimulus at time zero. By contrast, in the unpredictable condition there was no clue as to when the pain stimulus would be given. The unpredictable condition was represented by blue triangles pointing upwards, in which the numbers counted upwards beginning with the number 1 , and ending on 3,6 , 9 , or 12 depending on the anticipatory period duration. Hence, in both conditions the anticipatory visual stimuli changed once every second until stimulus delivery. Our design therefore prevents attentional lapses that may occur during longer anticipatory periods when there is a relative lack of novel sensory input. We reasoned that such lapses in attention may inadvertently influencing pain responses. Such exogenous cues have in the past been used to maintain attention, showing measurable effects on attention areas in the brain (O'Connor et al., 2005).

Following the laser stimulus (given at the pre-determined level 7 in all trials) there was a 3 -s resting period, during which time participants were asked to remain still and focus on the screen ahead, awaiting the next prompt. This allowed EEG recording of the LEP. The participant was then prompted to rate the painfulness of the stimulus by the appearance of the $0-10$ pain scale on the computer screen. The experimental design is schematically represented in Fig. 1.

Each of the 8 trial conditions (i.e. the 4 anticipation durations $(3 \mathrm{~s}, 6 \mathrm{~s}, 9 \mathrm{~s}$, and $12 \mathrm{~s}) \times 2$ predictability conditions) was presented 20 times across 4 blocks lasting approximately 10 min each. Trials were presented in a pseudo-random order with each block containing 5 of each trial type. This ensured that each condition was evenly distributed through the experiment and prevented habituation interfering with condition effects. Subjects were made aware that the probability of receiving each condition was the same on each trial.

EEG recordings were taken from 61 scalp electrodes placed according to an extended 10-20 system (Easycap coupled with Neuroscan amplifiers). Electrodes were referenced to the ipsilateral (right) earlobe, and recordings were also taken from the contralateral (left) earlobe for off-line conversion to linked ears reference. 


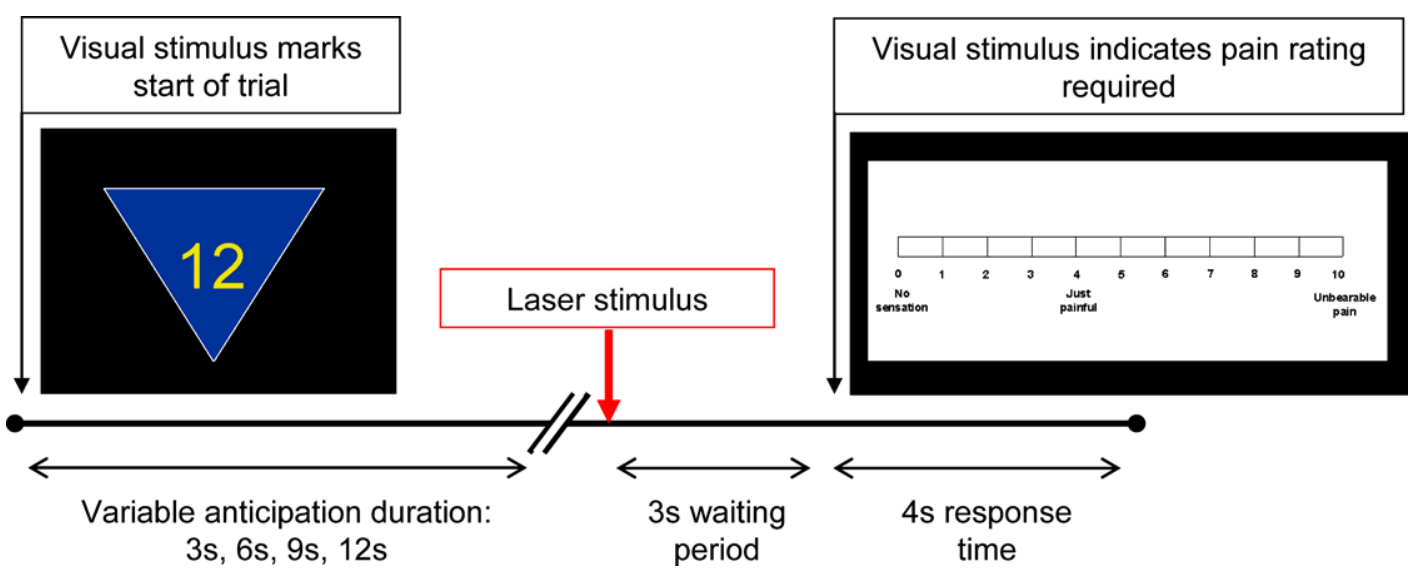

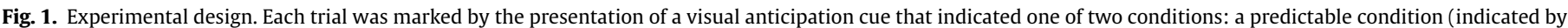

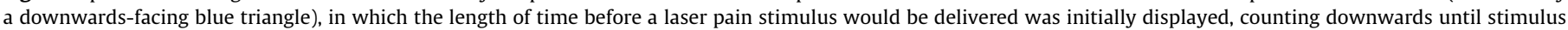

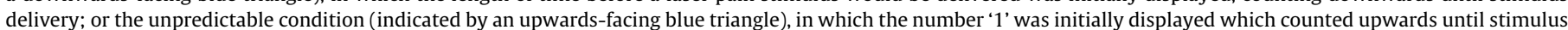

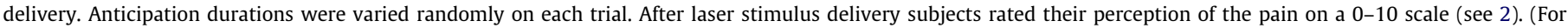
interpretation of color mentioned in this figure the reader is referred to the web version of the article.)

Electrode impedance was kept lower than $5 \mathrm{k} \Omega$. Two electro-oculographic (EOG) channels were used to monitor eye-movements and blinking, this allowed off-line reduction of blink and eyemovement artifacts. Bandpass filters were set at DC- $100 \mathrm{~Hz}$, with a sampling rate of $500 \mathrm{~Hz}$ and gain of 500 . A notch filter was set to $50 \mathrm{~Hz}$ to reduce electrical interference.

\subsection{Behavioral data analysis}

The behavioral data obtained from the subject's.s ratings of pain intensity were normalized by subtracting each subject's mean pain rating (across all conditions) from their ratings for each individual trial. This was done to correct for any variation between subjects resulting from different interpretations of the pain scale during the psychophysics procedure. No two participants are equal and their individual responses often demonstrate large variability. Measuring trial effects relative to the average response made by that subject (across all trials) allows subjects to serve as their own control. Thus, variability in the average response resulting from experimental error can then be eliminated (Reed, 2004). The resulting data was tested for normality before conducting a repeated measures ANOVA to determine the effects of subject, predictability, and anticipation duration on the normalized behavioral data. Post hoc paired $t$-tests were then used to determine where the main effects were in the results.

\subsection{Electroencephalographic (EEG) data analysis}

The EEG data were analyzed using Neuroscan Edit 4.3 (Compumedics USA Ltd.) and Matlab 7.1 (Mathworks Inc.). The data were filtered to remove slow potentials below $0.005 \mathrm{~Hz}$ and downsampled from $500 \mathrm{~Hz}$ to $125 \mathrm{~Hz}$. Independent component analysis (ICA) was used to remove vertical and horizontal eye movement artifacts, which has previously been shown to be a successful method in analyzing ERPs (Jung et al., 2000b; Jung et al., 2000a). The data was epoched into single trials from $500 \mathrm{~ms}$ before the first visual stimulus to $1500 \mathrm{~ms}$ after the painful stimulus. Epochs were visually inspected for further artifacts (e.g. excessive muscle activity) that had escaped ICA removal, and deleted if necessary. Linear trends over the whole epoch were removed using the entire epoch to calculate the linear component in all channels. Each epoch was then baseline corrected to the $500 \mathrm{~ms}$ interval preceding the first visual anticipation cue. Trials were then averaged separately for each condition. Data were ref- erenced to the common average before proceeding further with data analysis, although ERP waveforms are presented according to the linked ears reference.

The P2 component of the Laser-Evoked Potential (LEP) was analyzed. P2 latencies were determined at $\mathrm{Cz}$ (where the P2 was at maximum amplitude over the whole group) for each subject and condition. A 16-ms window of LEP data was then extracted centered on the subject-specific latency for each condition. The P2 peak amplitude was analyzed using a repeated measures ANOVA (with Greenhouse-Geisser corrections) that consisted of three factors: anticipation duration, unpredictability of stimulus timing and electrode. The electrodes used in this analysis (F7, F3, FZ, F4, F8, T7, FCZ, C3, CZ, C4, T8, CPZ, P7, P3, PZ, P4, P8, O1, O2) were selected to include the midline electrodes and provide a good distribution across the head. $\mathrm{FC}, \mathrm{Cz}$, and $\mathrm{CPz}$ electrodes were then selected for further repeated measures ANOVAs to look at the main effects of predictability and anticipation duration. These results were adjusted using Greenhouse-Geisser and corrected for multiple comparisons using Bonferroni's correction.

We further analyzed the data from electrode $\mathrm{CPz}$ by performing Pearson's correlations between the main effects of unpredictability of stimulus timing and anticipation duration on P2 amplitude. Specifically, we compared the main effect of P2 amplitudes for [unpredictable - predictable] conditions (averaged across the different anticipation durations) vs. the difference in P2 amplitudes for different anticipation durations in the unpredictable condition (e.g. [12s-9s], and [9s-3s]). These results were adjusted using Greenhouse-Geisser and corrected for multiple comparisons using Bonferroni's correction.

\subsection{Source analysis}

Sources of the P2 were estimated with low-resolution electromagnetic tomography (LORETA), using the LORETA-KEY software (Pascual-Marqui et al., 2002). There is considerable evidence to suggest that LORETA is accurate in localizing the sources of EEG data, even in some deeper structures. Work has shown substantial similarity between intra-cerebral recordings and source localization foci (Seeck et al., 1998; Trebuchon-Da Fonseca et al., 2005), including LORETA analyses of deep mesial temporal/hippocampal and subcallosal cingulate foci (Zumsteg et al., 2005). Moreover, in two EEG/fMRI studies LORETA localizations were, on average $16 \mathrm{~mm}$ (Mulert et al., 2004) and $14.5 \mathrm{~mm}$ (Vitacco et al., 2002) from fMRI activation loci, including loci buried in the depths of 


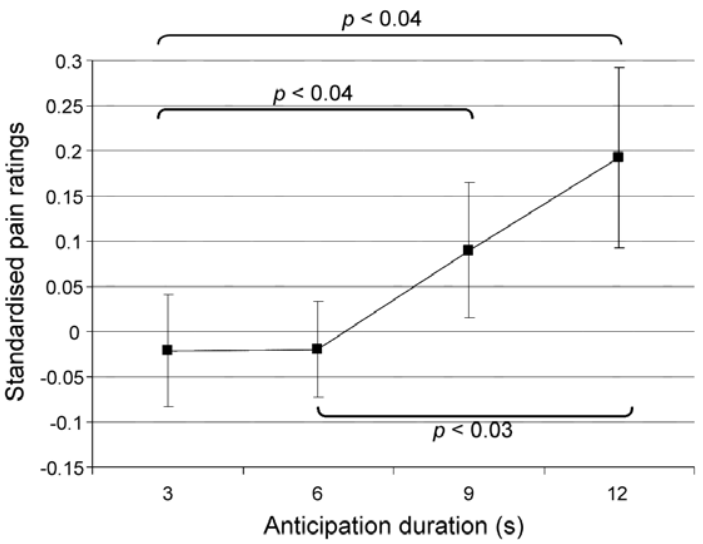

the insula. Similar results have been reported elsewhere (Benar et al., 2005).

The LORETA software uses a three-shell spherical head model registered to the Talairach anatomical brain atlas (Talairach and Tournoux, 1988), although the electrode coordinates used were determined from a co-registration between spherical and realistic head geometry that created a best-fit model (Towle et al., 1993). LORETA estimates ERP sources in grey matter volume to a Z$\mathrm{mm}^{3}$ grid resolution (2394 voxels in total) using the digitized MNI probability atlas (Mazziotta et al., 2001). Time-domain EEG files were converted to current density vector field magnitude using this technique. The resulting LORETA solutions were logtransformed at each voxel; this approximates LORETA solutions to a Gaussian distribution for parametric statistical analysis as previously demonstrated (Liu and Perfetti, 2003; Kiebel et al., 2005).

LORETA solutions were converted to SPM image format using a modified version of LOR2SPM (http://www.ihb.spb.ru/ pet_lab/ L2S/L2SMain.htm). During this process LORETA solutions were intensity-normalized in order to eliminate subject-to-subject global variations. Statistical maps were then created using SPM5 (http://www.fil.ion.ucl.ac.uk/spm/software/spm5) running on Matlab 7.1. The data were analyzed as follows. Firstly, the predictable and unpredictable conditions were compared (averaged across all anticipation durations) using paired samples $t$-tests. Secondly, the effect of increasing the anticipation duration was considered (with predictable and unpredictable averaged together) using an analysis in which the anticipation duration was entered as the covariate (i.e. 3, 6, 9, or 12). This showed areas of the brain more active after longer anticipatory period durations. Thirdly, a regression analysis was used to correlate brain activity with behavioral data. To do this we took each participant's average pain rating for trials with a 12-s anticipatory period duration and subtracted their average rating in the 3 -s trials. This gave us a measure of how much their pain ratings were affected by increasing the anticipatory period duration. Correlating this with LORETA data averaged over all conditions revealed which areas of the brain were more active in participants who were more affected by anticipation duration. Results were considered statistically significant at a threshold of $p<0.001$ (uncorrected) for areas known to mainly contribute to the amplitude of the P2 LEP peak (namely, midcingulate and insular/S2 cortices), and $p<0.05$ (FDR corrected) for all other areas, with 5 contiguous voxels above the threshold.

\section{Results}

\subsection{Behavioral data}

Pain ratings of the laser stimuli (Fig. 2) increased significantly as the duration of the anticipatory period increased $(F=5.14$, $p<0.003$ ). This effect occurred more in some participants than others: there was an interaction between anticipation duration and subject $(F=1.49, p<0.04)$. Post hoc paired t-tests revealed significant differences in pain ratings when making comparisons between $3 \mathrm{~s}$ and $9 \mathrm{~s}(t=2.40, p<0.04), 3 \mathrm{~s}$ and $12 \mathrm{~s}(t=2.30$, $p<0.04 \hat{)}$, and $6 \mathrm{~s}$ and $9 \mathrm{~s}(t=2.60, p<0.03 \hat{)}$ anticipation durations. There were no other main effects or interactions; notably there was no effect of unpredictability of stimulus timing on the pain ratings.

\subsection{EEG results}

The amplitude of the P2 was modulated by unpredictability $(F=13.1, p<0.004)$ and electrode $(F=23.9, p<0.001)$. Significant interaction effects were observed between unpredictability and electrode $(F=6.1, p<0.008)$, anticipation duration and electrode

Fig. 2. The effect of anticipation duration on pain perception. Pain ratings were normalized to each individual's mean pain rating and statistically compared between the four anticipation durations (averaged over predictable and unpredictable conditions). Longer anticipation durations induced significantly greater perception of pain.

$(F=2.4, p=0.046)$ and unpredictability, anticipation duration and electrode $F=2.8, p<0.03$ ). Topographic plots and waveform plots were drawn to visualize these effects (Fig. 3). The topographic plots show that the main effects of unpredictability were around the central midline electrodes, with greater unpredictability in the timing of the pain increasing $\mathrm{P} 2$ responses at electrodes $\mathrm{FCZ}$ $p<0.04), \mathrm{Cz}(p<0.03)$, and $\mathrm{CPz}(p<0.00)$.

The effect of unpredictability was considered in more detail at electrode $\mathrm{CPz}$, where the largest main effect of unpredictability was found. Fig. 4a shows that in the unpredictable condition there was a general increase in P2 amplitude from $3 \mathrm{~s}$ to $9 \mathrm{~s}$ with a decrease between $9 \mathrm{~s}$ and $12 \mathrm{~s}$. To consider whether the difference between the $9 \mathrm{~s}$ unpredictable trial and the $12 \mathrm{~s}$ unpredictable trial was due to a change in predictability we correlated this difference with the main effect of predictability on the P2 peak, i.e. the average difference (across all anticipation durations) between the predictable and unpredictable conditions. A significant correlation was found $(r=0.64, p<0.03)$. A correlation of $9 s-3 s$ in the unpredictable condition and the overall effect of predictability on the P2 was not significant.

The LORETA results are presented in Fig. 5 and Table 1 . The main effect of unpredictability showed greater activation in the secondary somatosensory cortex (S2) and posterior midcingulate cortex (MCC). Longer anticipation durations were associated with greater activation in bilateral posterior insulae, left anterior insula, right hippocampus/parahippocampal gyrus and right frontal pole, but not in the MCC. Activity in the anterior MCC correlated with the increase in pain ratings resulting from longer anticipation durations.

\section{Discussion}

In this study we sought to identify whether the duration of anticipation influences behavioral and brain responses to pain independently of the unpredictability of stimulus delivery time, whilst controlling for attention. Our behavioral results support the hypothesis that greater durations of anticipation increase pain perception, consistent with previous findings (Berns et al., 2006; Hauck et al., 2007). However, contrary to our expectations, the P2 amplitude did not vary with the duration of anticipation, but rather with unpredictability in stimulus timing. Instead, longer anticipation durations increased activation of bilateral insulae and hippocampal areas, whereas subjects whose pain ratings were the most influenced by anticipation duration showed greater acti- 


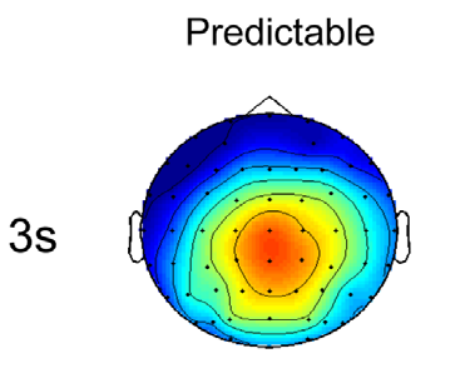

\section{Unpredictable}
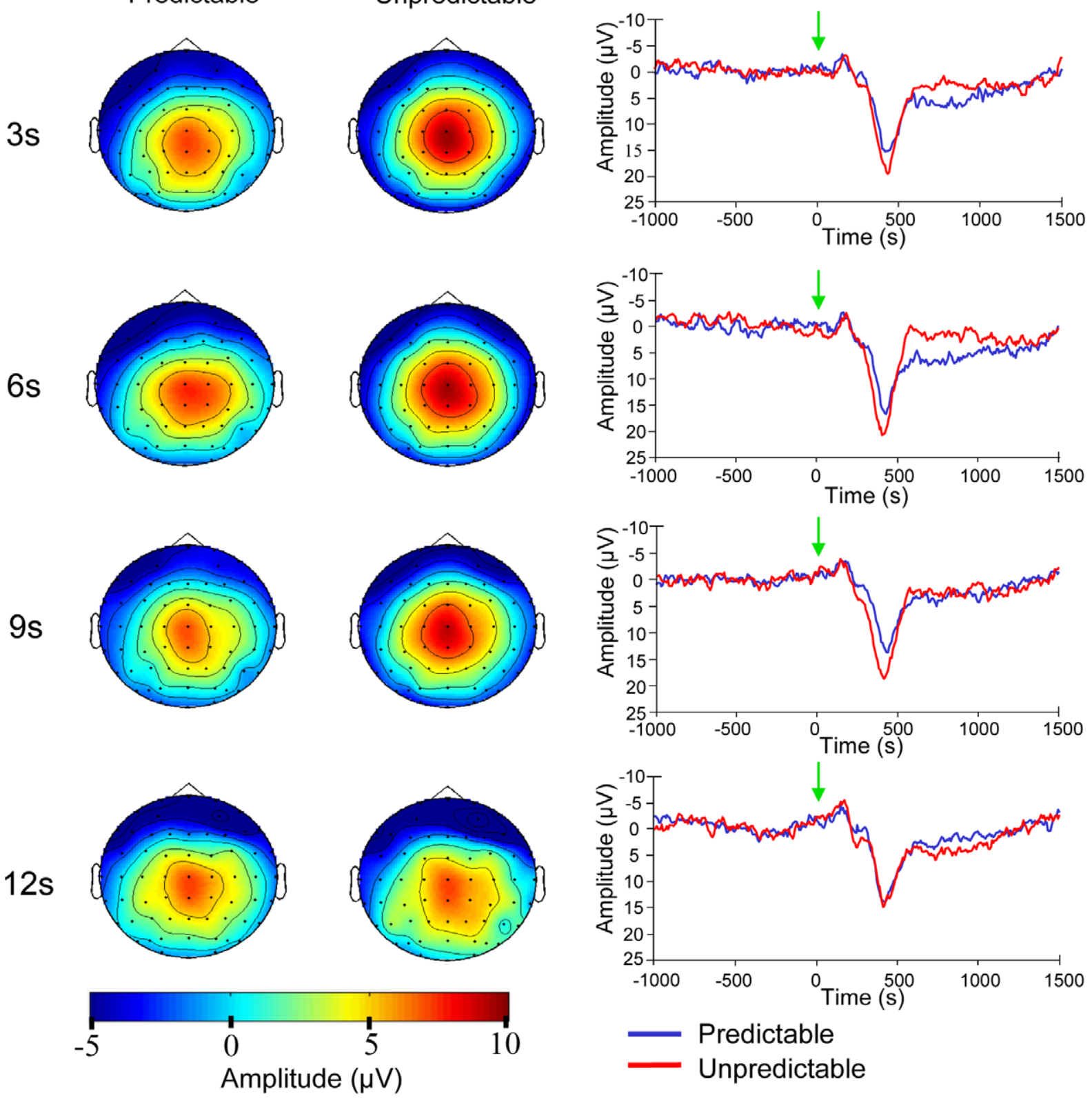

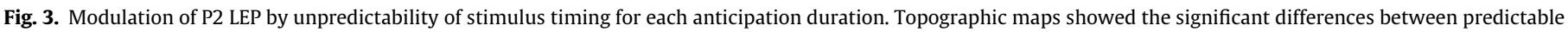

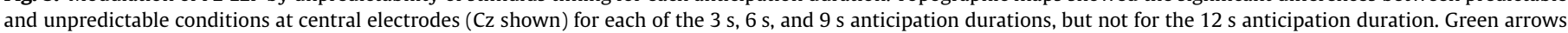
indicate onset of the laser stimulus. (For interpretation of color mentioned in this figure the reader is referred to the web version of the article.)

vation of anterior midcingulate cortex (MCC). These findings suggest a more specific network mediating the effects of anticipation duration on pain than previously thought.

There was no interaction between the unpredictability of stimulus timing and the duration of anticipation in the behavioral results, suggesting that unpredictability has little impact on subjective pain perception. However, unpredictability of stimulus timing increased the P2 of the pain-evoked response. This is consistent with previous results showing similar modulation of the P2 during oddball tasks when pain stimuli are novel (Legrain et al., 2002). We localized the sources of this modulation of the P2 to the MCC and ipsilateral secondary somatosensory cortex (S2), areas commonly identified as the major sources of the P2 (Iannetti et al., 2005; Bentley et al., 2002; Tarkka and Treede, 1993; Garcia-Larrea et al., 2003; Bentley et al., 2002; Valeriani et al., 2000). These areas have also been shown to be involved with attentional orientation to pain (Peyron et al., 1999; Peyron et al., 2000).

Previous studies have investigated the effects of increasing the duration of pain anticipation on subsequently perceived pain and its corresponding brain activity. A combined EEG and MEG study showed that longer anticipatory period durations were associated with larger P2 amplitudes (Hauck et al., 2007). However, the study did not separate out the possible effects of unpredictability of stimulus timing from anticipation duration as was performed in the current study. We also considered it important to control for possible changes in attention over time, which has not been done previously. For example, if subjects are not cued to maintain attention during pain anticipation, reduced attention would occur during longer anticipatory periods relative to shorter ones, resulting in greater attentional orientation to the pain stimulus once it arrives. Given that the P2 is generated from the MCC (Garcia-Larrea et al., 

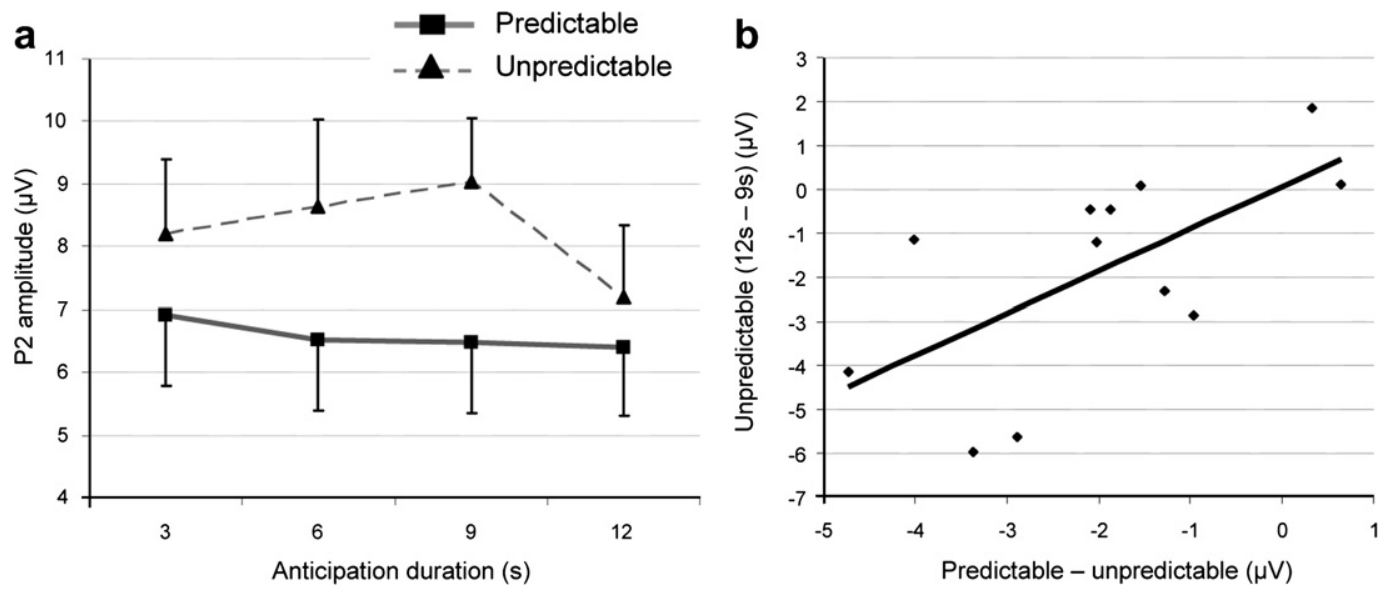

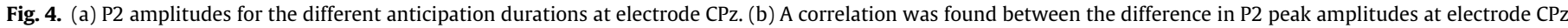

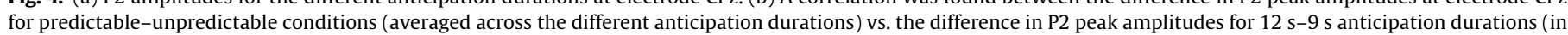
the unpredictable condition only).

2003), an area thought to represent attentional orientation towards a pain stimulus (Peyron et al., 1999), increased P2 amplitudes may occur with increasing anticipation duration as a result of changes in attention and not anticipation duration per se. Our paradigm attempted to control for attention by repeatedly presenting novel exogenous cues over the course of the anticipation period. A limitation of our study was that we did not take steps to monitor attention during the anticipation period. Adding in probes at random times during anticipation on some trials that require an immediate response, and then comparing reaction times, would have been fruitful in determining the effectiveness of repeated anticipation cues in maintaining attention. Even though we took no measures to measure attention, evidence suggests that the repeated presentation of exogenous cues improves the ability to sustain attention over time (O'Connor et al., 2005). We therefore regard this as an improvement on previous designs looking at anticipatory effects on the P2.

Contrary to previous data in which attention and unpredictability of stimulus timing were not controlled for, our results showed no modulation of the P2 by anticipation duration.

This also contrasts with our behavioral findings, where pain rating $\overline{\overline{\bar{\sigma}}}$ eased with anticipation duration. Our findings may be perhaps considered surprising given that the P2 is the most robust temporal component of the LEP, and would be expected to be modulated by anticipation duration along with pain ratings. However, we suggest that the effects of anticipation duration on the P2 in previous studies were an artifact of changes in attention over time, and that the neural generators of the P2 are more closely related to processing attentional orientation to pain than intensity coding of the stimulus.

An attentional, rather than intensity coding, role for the neural generators of the P2 has been previous suggested (Garcia-Larrea et al., 1997) and elaborated (Peyron et al., 2000). Evidence for this hypothesis is twofold: firstly, the P2 is mostly generated from the MCC, which has been shown not to be involved with intensity coding (Peyron et al., 1999; Casey et al., 2001; Derbyshire et al., 1997), but rather is likely to be involved with attentional orientation towards pain (Peyron et al., 1999). Secondly, we have shown in the present study that unpredictability of stimulus timing modulates the amplitude of the P2 and current density within the MCC, without affecting pain perception. Previous work has suggested that uncertainty is a modulator of attention, with greater uncertainty (or greater unpredictability of stimulus timing in this case) stimu- lating attention and learning (Dayan and Abbott, 2001; Brown et al. 2008). Hence there is theoretical basis for considering the relationship between P2 and unpredictability of stimulus timing to be a function of attention.

Further results from the current study present additional evidence that the neural processes which give rise to the P2 are more closely related to unpredictability or attention than to the painenhancing effects of anticipation duration. We showed that there was a drop in amplitude of the P2 in the unpredictable condition after the longest pain anticipation duration relative to the shorter ones. We reasoned this drop was probably caused by an increase in the predictability of the pain stimulus timing: for trials containing $12 \mathrm{~s}$ anticipatory periods the pain stimulus was effectively predictable. As confirmation of this, the drop in P2 amplitude from the $9 \mathrm{~s}$ to the $12 \mathrm{~s}$ anticipation duration correlated with the main effect of predictability on the P2 peak across subjects. In other words, the best explanation for the change in P2 amplitude as anticipation duration increased was a change in unpredictability of stimulus timing.

If greater anticipation durations increase pain ratings without affecting the attentional functions indexed by the P2 of the LEP, by what mechanism do longer anticipation durations influence pain perception? The results of our LORETA analysis, in which we co-varied sources of the P2 with anticipation duration, shed light on this issue. With increasing anticipation duration, greater current density was found in bilateral insulae and hippocampal areas. Consistent with the lack of effects of time on the P2, these areas are not commonly thought to be the major generators of the P2 (Garcia-Larrea et al., 2003), but there is nevertheless evidence for their involvement (Bentley et al., 2001; Valeriani et al., 2000) and they are important modulators of pain experience. In fact, the insulae are one of the most important regions of the brain for the intensity coding of a nociceptive stimulus (Ploghaus et al., 2001; Coghill et al., 1999; Casey et al., 2001; Derbyshire et al., 1997). Our observation of activation in hippocampal and parahippocampal areas is of interest considering the large body of evidence that these areas serve important mnemonic functions (Milner et al., 1998), and are thought to be closely related to anxiety (Gray and McNaughton, 2000). An insula-hippocampal network has been implicated in the exacerbation of pain by anxiety (Ploghaus et al., 2001), whereas lesions of the dorsal hippocampus have anxiolytic effects (McHugh et al., 2004). Previous studies have also identified memory functions for the right frontal pole, and specifically in coding for pro- 


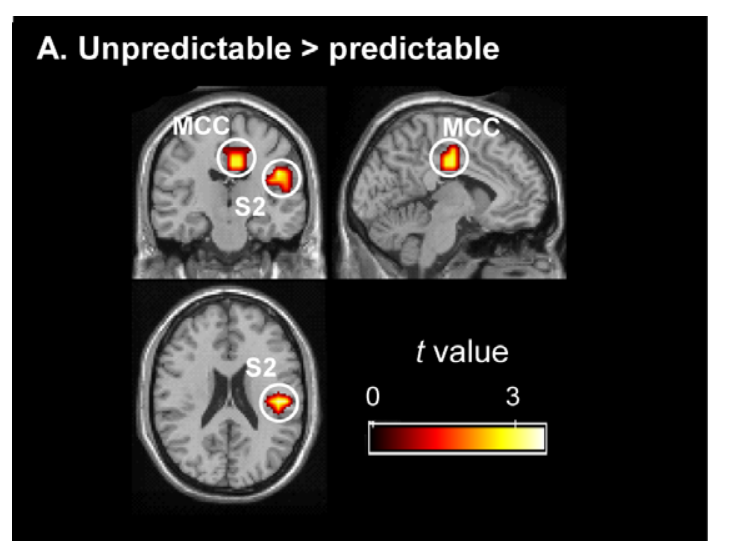

\section{B. Anticipation length (longer > shorter)}
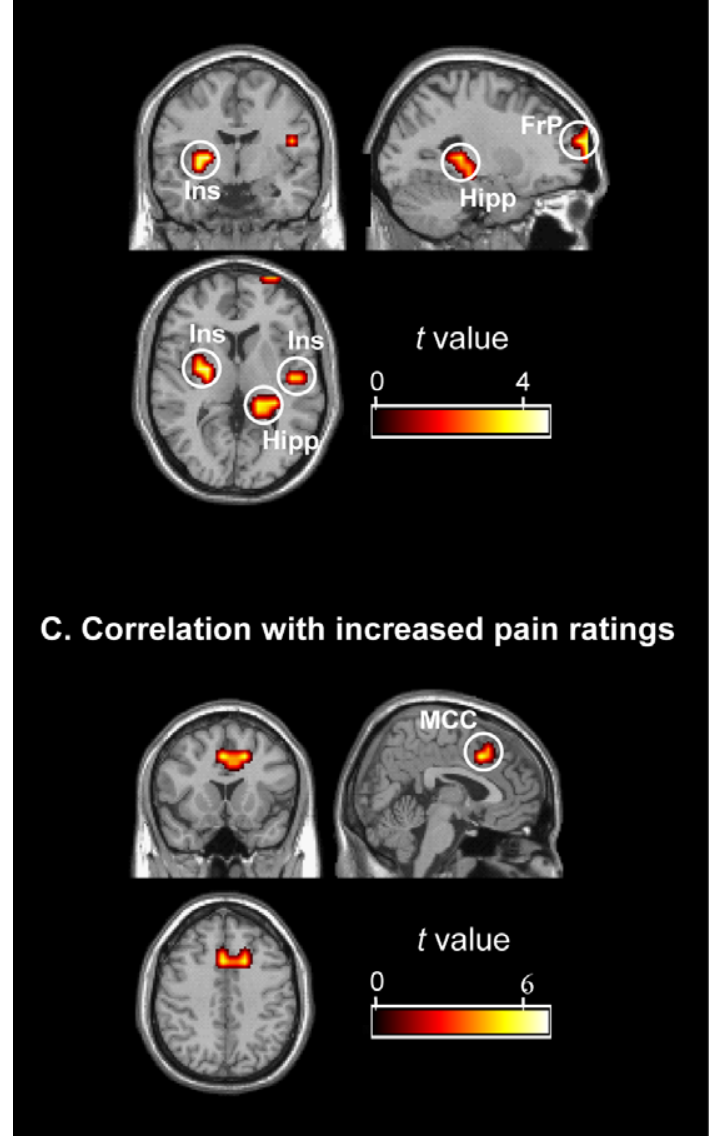

Midcingulate Cortex
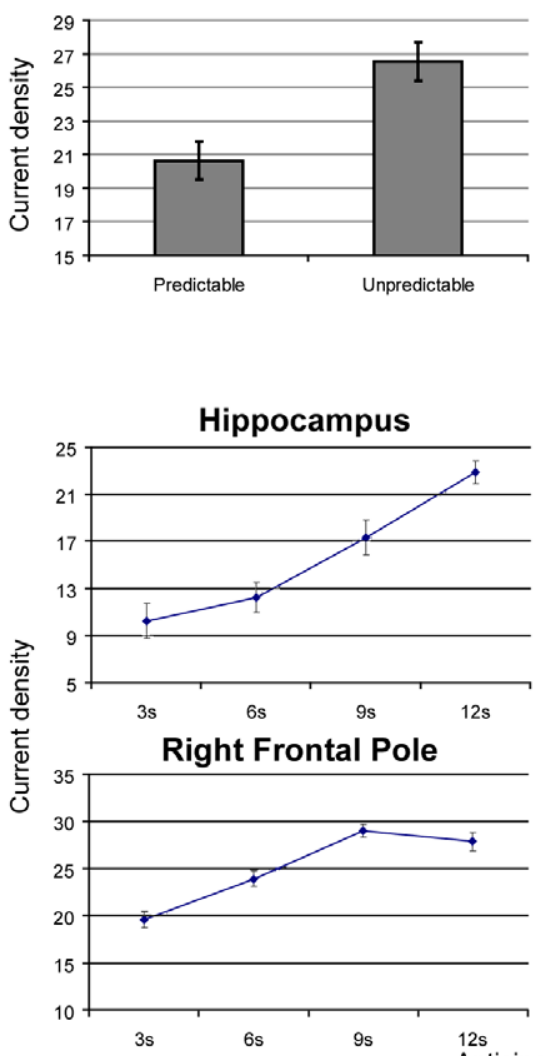

S2 Cortex

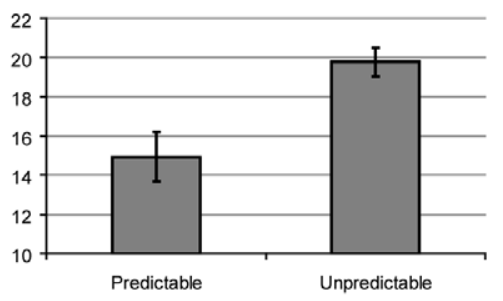

Left Insula
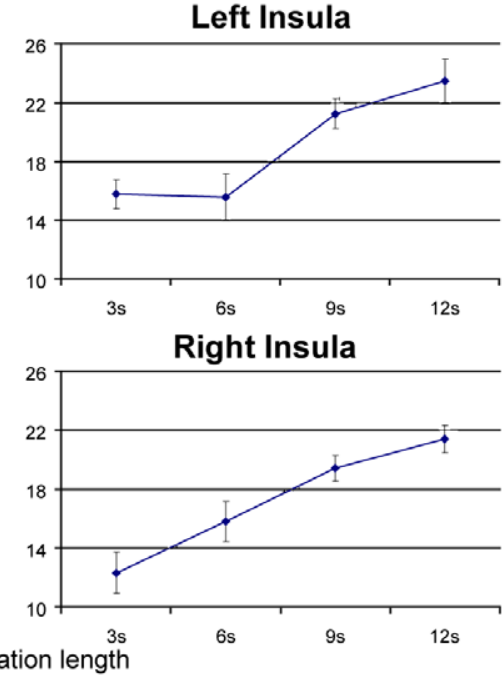

Midcingulate Cortex

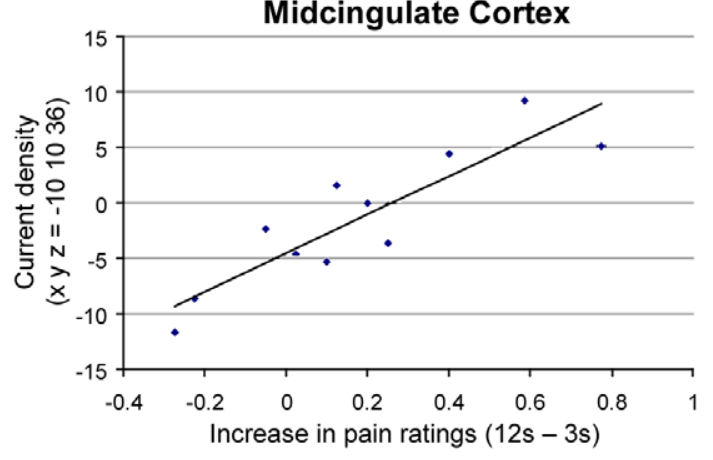

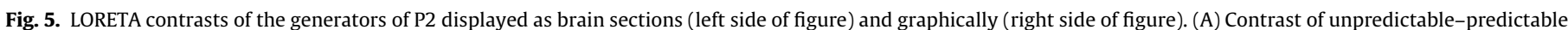

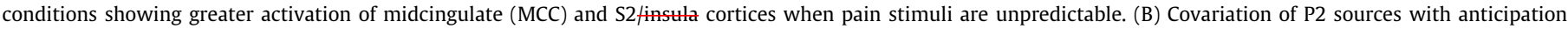

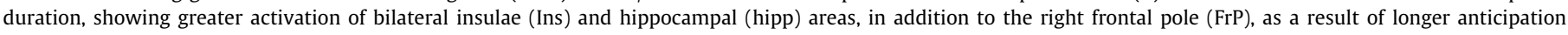

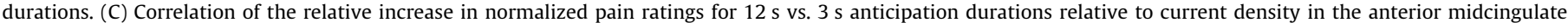
cortex (MCC). Error bars represent the standard error of the mean.

spective memory, i.e. memory for intended behaviors that will be realized in the future (Burgess et al., 2001; Okuda et al., 2003). The co-activation of insula cortices with (para)hippocampal areas and the right frontal pole suggests that longer anticipation periods may be augmenting pain perception via the activation of memory networks. Hence we suggest that anticipation duration affects the perception of pain via a hippocampal-insula-prefrontal cortical network, which operates independently of the degree of attentional orientation towards the stimulus (mediated by the midcingulate cortex).

A further LORETA analysis showed that anterior MCC (i.e. a region more anterior to the MCC generator of the P2) was activated more in subjects whose pain ratings were most influenced by the anticipation duration. This is consistent with previous work, in which 'extreme dreaders' (i.e. people who find it the most unpleasant to wait longer anticipation durations preceding pain) showed greater anticipatory increases in activity in the midcingulate cortex relative to 'mild dreaders' (Berns et al., 2006). It has been noted that the more posterior regions of MCC do not have affective functions and are likely to be more involved with skeleto-motor orienting, whereas the more anterior regions are responsive to affective influences (Vogt, 2005). In particular, the anterior MCC is a region known to be important for processing pain unpleasantness (Tolle et al., 1999; Kulkarni et al., 2005; Rainville et al., 1999), in addition 
Table 1

Brain regions showing variations in current density with unpredictability, pain anticipation duration, and anticipatory enhancement of pain perception.

\begin{tabular}{|c|c|c|c|c|c|c|c|}
\hline \multirow[t]{2}{*}{ Brain region } & & \multirow[t]{2}{*}{ Area } & \multicolumn{3}{|c|}{ MNI coordinates } & \multirow[t]{2}{*}{$t$ value } & \multirow[t]{2}{*}{$p$ value (Uncorr) } \\
\hline & & & $\mathbf{x}$ & $\mathbf{y}$ & $\mathbf{z}$ & & \\
\hline \multicolumn{8}{|c|}{ Main effect of unpredictability } \\
\hline S2/insular cortex & $\mathrm{R}$ & 13 & 39 & -25 & 22 & 3.53 & 0.000 \\
\hline Midcingulate cortex & $\mathrm{L}$ & 24 & -3 & -18 & 36 & 3.11 & 0.001 \\
\hline \multicolumn{8}{|c|}{ Main effect of longer pain anticipation } \\
\hline \multirow[t]{2}{*}{ Frontal pole } & $\mathrm{R}$ & 10 & 25 & 66 & 8 & 4.90 & 0.000 \\
\hline & $\mathrm{R}$ & 10 & 11 & 66 & 22 & 4.32 & 0.000 \\
\hline \multirow[t]{2}{*}{ Posterior insula } & $\mathrm{L}$ & 21 & -38 & -11 & -6 & 4.81 & 0.000 \\
\hline & $\mathrm{R}$ & 13 & 39 & -18 & 1 & 4.03 & 0.000 \\
\hline Anterior insula & $\mathrm{L}$ & 13 & -31 & 10 & 8 & 3.53 & 0.000 \\
\hline Hippocampus & $\mathrm{R}$ & & 25 & -39 & 1 & 3.99 & 0.000 \\
\hline \multirow[t]{2}{*}{ Parahippocampal gyrus } & $\mathrm{R}$ & 30 & 11 & -39 & 1 & 3.91 & 0.000 \\
\hline & $\mathrm{R}$ & 35 & 18 & -32 & -13 & 3.78 & 0.000 \\
\hline \multicolumn{8}{|c|}{ Correlation with increased pain ratings } \\
\hline \multirow[t]{2}{*}{ Midcingulate cortex } & $\mathrm{L}$ & 32 & -10 & 10 & 36 & 6.96 & 0.000 \\
\hline & $\mathrm{R}$ & 32 & 11 & 10 & 36 & 6.85 & 0.000 \\
\hline
\end{tabular}

Uncorr, uncorrected $P$ values. Area, Brodmann's area.

to anticipatory and pain-related anxiety (Kalisch et al., 2005; Simpson et al., 2001; Ploghaus et al., 2001). We therefore suggest that participants who experienced the greatest dread were susceptible to cognitive enhancement of pain perception via an independent mechanism from the anxiety induced by the hippocampal-insulaprefrontal network. The nature of this cognitive enhancement, however, is unclear. One possibility, suggested previously (Berns et al., 2006), is that greater dread enhances attention to pain. However, we must be careful when discussing attention to consider how pain processing can be modified by placing attention on different pain components and different points in time. Activation of anterior midcingulate is consistent with a greater attentional focus on the affective component of pain (Raij et al., 2005; Derbyshire et al., 2004; Rainville et al., 1997; Kulkarni et al., 2005), and possibly a future-oriented rather than present-oriented focus (Ploghaus et al., 2003; Ploghaus et al., 1999). This is in contrast to posterior midcingulate activations that can be best characterized by an attentional orientation to present stimuli (exemplified by the P2), which is not directly involved with affective functions. Hence we would speculate that 'extreme dreaders' have a more future-oriented and/or emotionally-focused style of attention.

To conclude, the present study demonstrates that the increase in perceived pain resulting from greater anticipation durations are not related to changes in unpredictability of stimulus timing or attention, and are mediated by a hippocampal-insula-prefrontal network. Unpredictability in the timing of pain is likely to increase attentional orientation to pain without any corresponding increase in pain perception, via midcingulate and S2 cortex. More anterior regions of midcingulate cortex may further enhance the effects of longer anticipation durations in individuals who are susceptible to experiencing dread.

\section{Acknowledgements}

This work was funded by the University of Manchester and the Arthritis Research Campaign.

\section{References}

Bantick SJ, Wise RG, Ploghaus A, Clare S, Smith SM, Tracey I. Imaging how attention modulates pain in humans using functional MRI. Brain 2002;125:310-9.

Benar CG, Gunn RN, Grova C, Champagne B, Gotman J. Statistical maps for EEG dipolar source localization. IEEE Trans Biomed Eng 2005;52:401-13.

Bentley DE, Derbyshire SW, Youell PD, Jones AK. Caudal cingulate cortex involvement in pain processing: an inter-individual laser evoked potential source localisation study using realistic head models. Pain 2003;102:265-71.
Bentley DE, Youell PD, Crossman AR, Jones AK. Source localisation of 62-electrode human laser pain evoked potential data using a realistic head model. Int J Psychophysiol 2001;41:187-93.

Bentley DE, Youell PD, Jones AK. Anatomical localization and intra-subject reproducibility of laser evoked potential source in cingulate cortex, using a realistic head model. Clin Neurophysiol 2002;113:1351-6.

Berns GS, Chappelow J, Cekic M, Zink CF, Pagnoni G, Martin-Skurski ME. Neurobiological substrates of dread. Science 2006;312:754-8.

Brown CA, Jones AKP. A role for midcingulate cortex in the interruptive effects of pain anticipation on attention. Clin Neurophysiol, in press.

Brown CA, Seymour B, Boyle Y, El-Deredy W, Jones AKP. Modulation of pain perception by expectation and uncertainty: behavioral characteristics and anticipatory neural correlates. Pain 2008;135:240-50.

Brown CA, Seymour B, El-Deredy W, Jones AKP. Confidence in beliefs about pain predicts expectancy effects on pain perception and anticipatory processing in right anterior insula. Pain, in press.

Buffington AL, Hanlon CA, McKeown MJ. Acute and persistent pain modulation of attention-related anterior cingulate fMRI activations. Pain 2005;113:172-84.

Burgess PW, Quayle A, Frith CD. Brain regions involved in prospective memory as determined by positron emission tomography. Neuropsychologia 2001;39:545-55.

Caplin A, Leahy J. Psychological expected utility theory and anticipatory feelings. Quart J Econ 2001;116:55-79.

Carlsson K, Andersson J, Petrovic P, Petersson KM, Ohman A, Ingvar M. Predictability modulates the affective and sensory-discriminative neural processing of pain Neuroimage 2006;32:1804-14.

Casey KL, Morrow TJ, Lorenz J, Minoshima S. Temporal and spatial dynamics of human forebrain activity during heat pain: analysis by positron emission tomography. J Neurophysiol 2001;85:951-9.

Coghill RC, Sang CN, Maisog JM, Iadarola MJ. Pain intensity processing within the human brain: a bilateral, distributed mechanism. J Neurophysiol 1999;82:1934-43.

Craig AD. A new view of pain as a homeostatic emotion. Trends Neurosci 2003;26:303-7.

Dayan P, Abbott LF. Theoretical neuroscience: Computational and mathematical modeling of neural systems 2001. MIT Press.

Derbyshire SW, Jones AK, Gyulai F, Clark S, Townsend D, Firestone LL. Pain processing during three levels of noxious stimulation produces differential patterns of central activity. Pain 1997;73:431-45.

Derbyshire SW, Whalley MG, Stenger VA, Oakley DA. Cerebral activation during hypnotically induced and imagined pain. Neuroimage 2004;23:392-401.

Garcia-Larrea L, Frot M, Valeriani M. Brain generators of laser-evoked potentials: from dipoles to functional significance. Neurophysiol Clin 2003;33:279-92.

Garcia-Larrea L, Peyron R, Laurent B, Mauguiere F. Association and dissociation between laser-evoked potentials and pain perception. Neuroreport 1997;8:3785-9.

Gray JA, McNaughton N. The neuropsychology of anxiety: An enquiry into the functions of the Septo-Hippocampal system. Oxford: Oxford University Press; 2000.

Hauck M, Lorenz J, Zimmermann R, Debener S, Scharein E, Engel AK. Duration of the cue-to-pain delay increases pain intensity: a combined EEG and MEG study. Exp Brain Res 2007;180:205-15.

Iannetti GD, Niazy RK, Wise RG, Jezzard P, Brooks JC, Zambreanu L, et al Simultaneous recording of laser-evoked brain potentials and continuous, high-field functional magnetic resonance imaging in humans. Neuroimage 2005;28:708-19.

Jones AK, Kulkarni B, Derbyshire SW. Pain mechanisms and their disorders. Br Med Bull 2003;65:83-93. 
Jung TP, Makeig S, Humphries C, Lee TW, McKeown MJ, Iragui V, et al. Removing electroencephalographic artifacts by blind source separation. Psychophysiology 2000a;37:163-78.

Jung TP, Makeig S, Westerfield M, Townsend J, Courchesne E, Sejnowski TJ. Removal of eye activity artifacts from visual event-related potentials in normal and clinical subjects. Clin Neurophysiol 2000b;111:1745-58.

Kalisch R, Wiech K, Critchley HD, Seymour B, O’Doherty JP, Oakley DA, et al. Anxiety reduction through detachment: subjective, physiological, and neural effects. J Cogn Neurosci 2005;17:874-83.

Keefe FJ, Rumble ME, Scipio CD, Giordano LA, Perri LM. Psychological aspects of persistent pain: current state of the science. J Pain 2004;5:195-211.

Kiebel SJ, Tallon-Baudry C, Friston KJ. Parametric analysis of oscillatory activity as measured with EEG/MEG. Hum Brain Mapp 2005;26:170-7.

Kulkarni B, Bentley DE, Elliott R, Youell P, Watson A, Derbyshire SW, et al. Attention to pain localization and unpleasantness discriminates the functions of the medial and lateral pain systems. Eur J Neurosci 2005;21:3133-42.

Legrain V, Guerit JM, Bruyer R, Plaghki L. Attentional modulation of the nociceptive processing into the human brain: selective spatial attention, probability of stimulus occurrence, and target detection effects on laser evoked potentials. Pain 2002;99:21-39.

Liu Y, Perfetti CA. The time course of brain activity in reading English and Chinese: an ERP study of Chinese bilinguals. Hum Brain Mapp 2003;18:167-75.

Mazziotta J, Toga A, Evans A, Fox P, Lancaster J, Zilles K, et al. A probabilistic atlas and reference system for the human brain: International Consortium for Brain Mapping (ICBM). Philos Trans R Soc Lond B Biol Sci 2001;356:1293-322.

McCaul KD, Haugtvedt C. Attention, distraction, and cold-pressor pain. J Pers Soc Psychol 1982;43:154-62.

McHugh SB, Deacon RMJ, Rawlins JNP, Bannerman DM. Amygdala and ventral hippocampus contribute differentially to mechanisms of fear and anxiety. Behav Neurosci 2004;118:63-78.

Melzack R. Pain and the neuromatrix in the brain. J Dent Educ 2001;65:1378-82.

Melzack R, Wall PD. Pain mechanisms: a new theory. Science 1965;150:971-9.

Miller SM. Predictability and human stress: toward a clarification of evidence and theory. Adv Exp Soc Psychol 1981;14:203.

Milner B, Squire LR, Kandel ER. Cognitive neuroscience and the study of memory. Neuron 1998;20:445-68.

Mulert C, Jager L, Schmitt R, Bussfeld P, Pogarell O, Moller HJ, et al. Integration of fMRI and simultaneous EEG: towards a comprehensive understanding of localization and time-course of brain activity in target detection. Neuroimage 2004;22:83-94.

O’Connor C, Manly T, Robertson IH, Hevenor SJ, Levine B. An fMRI study of sustained attention with endogenous and exogenous engagement. Brain Cogn 2005;54:133-76.

Okuda J, Fujii T, Ohtake H, Tsukiura T, Tanji K, Suzuki K, et al. Thinking of the future and past: the roles of the frontal pole and the medial temporal lobes. Neuroimage 2003;19:1369-80.

Pascual-Marqui RD, Esslen M, Kochi K, Lehmann D. Functional imaging with lowresolution brain electromagnetic tomography (LORETA): a review. Methods Find Exp Clin Pharmacol 2002;24(Suppl. C):91-5.

Peyron R, Garcia-Larrea L, Gregoire MC, Costes N, Convers P, Lavenne F, et al. Haemodynamic brain responses to acute pain in humans: sensory and attentional networks. Brain 1999;122(Pt. 9):1765-80.

Peyron R, Laurent B, Garcia-Larrea L. Functional imaging of brain responses to pain. A review and meta-analysis. Neurophysiol Clin 2000;30:263-88.
Ploghaus A, Becerra L, Borras C, Borsook D. Neural circuitry underlying pain modulation: expectation, hypnosis, placebo. Trends Cogn Sci 2003;7:197-200.

Ploghaus A, Narain C, Beckmann CF, Clare S, Bantick S, Wise R, et al. Exacerbation of pain by anxiety is associated with activity in a hippocampal network. J Neurosci 2001:21:9896-903.

Ploghaus A, Tracey I, Gati JS, Clare S, Menon RS, Matthews PM, et al. Dissociating pain from its anticipation in the human brain. Science 1999;284:1979-81.

Price DD. Psychological and neural mechanisms of the affective dimension of pain. Science 2000;288:1769-72.

Raij TT, Numminen J, Narvanen S, Hiltunen J, Hari R. Brain correlates of subjective reality of physically and psychologically induced pain. Proc Natl Acad Sci USA 2005:102:2147-51.

Rainville P, Carrier B, Hofbauer RK, Bushnell MC, Duncan GH. Dissociation of sensory and affective dimensions of pain using hypnotic modulation. Pain 1999;82:159-71.

Rainville P, Duncan GH, Price DD, Carrier B, Bushnell MC. Pain affect encoded in human anterior cingulate but not somatosensory cortex. Science 1997;277:968-71.

Reed III JF. Analysis of variance (ANOVA) models in emergency medicine. Internet J Emerg Intens Care Med 2004:7.

Seeck M, Lazeyras F, Michel CM, Blanke O, Gericke CA, Ives J, et al. Non-invasive epileptic focus localization using EEG-triggered functional MRI and electromagnetic tomography. Electroencephalogr Clin Neurophysiol 1998;106:508-12.

Simpson Jr JR, Drevets WC, Snyder AZ, Gusnard DA, Raichle ME. Emotion-induced changes in human medial prefrontal cortex: II. During anticipatory anxiety. Proc Natl Acad Sci USA 2001;98:688-93.

Talairach J, Tournoux P. Co-planar stereotaxic Atlas of the human brain. New York: Thieme; 1988.

Tarkka IM, Treede RD. Equivalent electrical source analysis of pain-related somatosensory evoked potentials elicited by a CO2 laser. J Clin Neurophysiol 1993;10:513-9.

Tolle TR, Kaufmann T, Siessmeier T, Lautenbacher S, Berthele A, Munz F, et al. Region-specific encoding of sensory and affective components of pain in the human brain: a positron emission tomography correlation analysis. Ann Neurol 1999;45:40-7.

Towle VL, Bolanos J, Suarez D, Tan K, Grzeszczuk R, Levin DN, et al. The spatial location of EEG electrodes: locating the best-fitting sphere relative to cortical anatomy. Electroencephalogr Clin Neurophysiol 1993;86:1-6.

Trebuchon-Da Fonseca A, Giraud K, Badier JM, Chauvel P, Liegeois-Chauvel C. Hemispheric lateralization of voice onset time (VOT) comparison between depth and scalp EEG recordings. Neuroimage 2005;27:1-14.

Valeriani M, Restuccia D, Barba C, Le Pera D, Tonali P, Mauguiere F. Sources of cortical responses to painful $\mathrm{CO}(2)$ laser skin stimulation of the hand and foot in the human brain. Clin Neurophysiol 2000;111:1103-12.

Vitacco D, Brandeis D, Pascual-Marqui R, Martin E. Correspondence of event-related potential tomography and functional magnetic resonance imaging during language processing. Hum Brain Mapp 2002;17:4-12.

Vogt BA. Pain and emotion interactions in subregions of the cingulate gyrus. Nat Rev Neurosci 2005;6:533-44.

Zumsteg D, Friedman A, Wennberg RA, Wieser HG. Source localization of mesial temporal interictal epileptiform discharges: correlation with intracranial foramen ovale electrode recordings. Clin Neurophysiol 2005;116:2810-8. 